\title{
A doença de Alzheimer: aspectos fisiopatológicos e farmacológicos
}

\section{Alzheimer's disease: pathophysiological and pharmacological features}

\author{
Adriana Sereniki'; Maria Aparecida Barbato Frazão Vital" I \\ IMestre em Farmacologia. \\ II Professora, Setor de Ciências Biológicas, Departamento de Farmacologia, Universidade Federal \\ do Paraná (UFPR), Curitiba, PR.
}

Endereço para correspondência

Rev Psiquiatr RS. 2008;30(1 Supl).

\section{RESUMO}

A doença de Alzheimer é a patologia neurodegenerativa mais freqüente associada à idade, cujas manifestações cognitivas e neuropsiquiátricas resultam em deficiência progressiva e incapacitação. A doença afeta aproximadamente $10 \%$ dos indivíduos com idade superior a 65 anos e $40 \%$ acima de 80 anos. Estima-se que, em 2050, mais de $25 \%$ da população mundial será idosa, aumentando, assim, a prevalência da doença. O sintoma inicial da doença é caracterizado pela perda progressiva da memória recente. Com a evolução da patologia, outras alterações ocorrem na memória e na cognição, entre elas as deficiências de linguagem e nas funções vísuo-espaciais. Esses sintomas são freqüentemente acompanhados por distúrbios comportamentais, incluindo agressividade, depressão e alucinações. O objetivo deste trabalho foi revisar, na literatura médica, os principais aspectos que envolvem a doença de Alzheimer, como as características histopatológicas, a neuroinflamação e a farmacoterapia atual.

Descritores: Doença de Alzheimer, inflamação, acetilcolinesterase, inibidores de ciclooxigenase.

\section{ABSTRACT}

Alzheimer's disease is the most frequent age-associated neurodegenerative disease. Its 
cognitive and neuropsychiatric manifestations result in progressive disorder and disability. Alzheimer's disease affects approximately $10 \%$ of patients more than 65 years old and $40 \%$ of patients more than 80 years old. It is estimated that, in $2050,25 \%$ of the global population will be elderly, thus increasing the disease prevalence. The initial symptom is characterized by progressive loss of recent memory. The progressive impairment in cognitive faculties such as memory, verbal and visuospatial ability is often accompanied by behavioral disorders, such as aggressiveness, depression and hallucinations. This article aims at reviewing the main aspects in Alzheimer's disease, such as histopathologic features, neuroinflammation and current pharmacotherapy.

Keywords: Alzheimer's disease, inflammation, acetylcholinesterase, cyclooxygenase inhibitors.

\section{NTRODUÇÃO}

A doença de Alzheimer é a patologia neurodegenerativa mais freqüente associada à idade, cujas manifestações cognitivas e neuropsiquiátricas resultam em uma deficiência progressiva e uma eventual incapacitação ${ }^{1,2}$. Em geral, o primeiro aspecto clínico é a deficiência da memória recente, enquanto as lembranças remotas são preservadas até um certo estágio da doença. Além das dificuldades de atenção e fluência verbal, outras funções cognitivas deterioram à medida que a patologia evolui, entre elas a capacidade de fazer cálculos, as habilidades vísuoespaciais e a capacidade de usar objetos comuns e ferramentas ${ }^{3}$. O grau de vigília e a lucidez do paciente não são afetados até a doença estar muito avançada. A fraqueza motora também não é observada, embora as contraturas musculares sejam uma característica quase universal nos estágios avançados da patologia ${ }^{3}$.

Esses sintomas são freqüentemente acompanhados por distúrbios comportamentais, como agressividade, alucinações, hiperatividade, irritabilidade e depressão ${ }^{4}$. Transtornos do humor afetam uma porcentagem considerável de indivíduos que desenvolvem doença de Alzheimer, em algum ponto da evolução da síndrome demencial ${ }^{4}$. Sintomas depressivos são observados em até $40-50 \%$ dos pacientes, enquanto transtornos depressivos acometem em torno de 10$20 \%$ dos $\operatorname{casos}^{6}$. Outros sintomas, como a apatia, a lentificação (da marcha ou do discurso), a dificuldade de concentração, a perda de peso, a insônia e a agitação podem ocorrer como parte da síndrome demencial? .

\section{FISI OPATOLOGI A DA DOENÇA DE ALZHEI MER}

A doença de Alzheimer caracteriza-se, histopatologicamente, pela maciça perda sináptica e pela morte neuronal observada nas regiões cerebrais responsáveis pelas funções cognitivas, incluindo o córtex cerebral, o hipocampo, o córtex entorrinal e o estriado ventral ${ }^{8}$.

As características histopatológicas presentes no parênquima cerebral de pacientes portadores da doença de Alzheimer incluem depósitos fibrilares amiloidais localizados nas paredes dos vasos sangüíneos, associados a uma variedade de diferentes tipos de placas senis, acúmulo de filamentos anormais da proteína tau e conseqüente formação de novelos neurofibrilares (NFT), perda neuronal e sináptica, ativação da glia e inflamação ${ }^{8}$.

Baseadas nesses marcadores neuropatológicos, duas hipóteses principais foram propostas, a fim de explicar a etiologia da doença. De acordo com a hipótese da cascata amiloidal, a neurodegeneração na doença de Alzheimer inicia-se com a clivagem proteolítica da proteína 
precursora amilóide (APP) e resulta na produção, agregação e deposição da substância $\beta$ amilóide $(A \beta)$ e placas senis ${ }^{9}$. De acordo com a hipótese colinérgica, a disfunção do sistema colinérgico é suficiente para produzir uma deficiência de memória em modelos animais, a qual é semelhante à doença de Alzheimer ${ }^{10}$. Cérebros de pacientes portadores da doença de Alzheimer mostraram degeneração dos neurônios colinérgicos ${ }^{11}$, ocorrendo também uma redução dos marcadores colinérgicos, sendo que a colina acetiltransferase e a acetilcolinesterase tiveram sua atividade reduzida no córtex cerebral de pacientes portadores da doença de Alzheimer ${ }^{11}$ (Tabela 1).

\begin{tabular}{|c|c|c|c|}
\hline Hipótese & Mecanismo molecular & Mecanismo secundário & Efeito final \\
\hline Hipótese da cascata amiloidal & $\begin{array}{l}\text { Clivagem proteolítica anormal } \\
\text { da APP } \\
\text { Produção, agregação e } \\
\text { deposição de placas senis } \\
\text { extracelulares } \\
\text { Formação de novelos } \\
\text { neurofibrilares intracelulares }\end{array}$ & $\begin{array}{l}\text { Produção de radicais livres, } \\
\text { ativaçấo da glia e inflamaçăo }\end{array}$ & Morte celular \\
\hline Hipótese colinérgica & $\begin{array}{l}\text { Redução da atividade da } \\
\text { colina acetiltransferase } \\
\text { Redução da atividade da } \\
\text { acetilcolinesterase }\end{array}$ & $\begin{array}{l}\text { Redução dos niveis de acetilcolina } \\
\text { Redućão na transmissão } \\
\text { colinérgica }\end{array}$ & Prejuizo cognitivo \\
\hline
\end{tabular}

Na maioria das células, a fosfolipase A2 (PLA2) contribui para a liberação do ácido araquidônico das membranas de fosfolipídeos, o qual é passo fundamental na síntese dos principais mediadores da resposta inflamatória ${ }^{12}$. Como a fosfatidilcolina é um dos substratos da PLA2, a redução da atividade dessa enzima poderia produzir um declínio no catabolismo da fosfatidilcolina, reduzindo a colina para a síntese de acetilcolina, contribuindo ainda mais para a deficiência colinérgica na doença de Alzheimer ${ }^{13}$. Em cérebros de pacientes portadores da doença de Alzheimer, a redução da atividade da acetilcolinesterase no córtex frontal e parietal foi relacionada ao início da demência, à quantidade de placas senis e NFT e à morte precoce desses pacientes ${ }^{14}$. Assim, a redução da atividade da PLA2 em pacientes portadores da doença de Alzheimer está diretamente relacionada à severidade da demência e ao grau do prejuízo cognitivo ${ }^{12}$.

A redução da atividade dessa enzima não foi relacionada ao tratamento com inibidores da acetilcolinesterase ou antipsicóticos ${ }^{12}$. As drogas antipsicóticas são conhecidas por reduzir a atividade da PLA $2^{15}$, porém a redução dessa enzima em cérebros de pacientes portadores da doença de Alzheimer permaneceu após a suspensão desses medicamentos ${ }^{12}$.

A hipótese da cascata amiloidal tem recebido suporte a partir de estudos genéticos com casos da forma familiar da doença de Alzheimer, nos quais mutações tanto na APP quanto nas presenilinas (PS) têm mostrado aumento na produção da substância $A \beta^{9}$. No entanto, enquanto o estudo dos casos da doença de Alzheimer familiar tem mostrado ser significativo para a compreensão dessa forma da patologia, as razões para a vulnerabilidade neuronal dos casos esporádicos da doença de Alzheimer permanecem desconhecidas ${ }^{16}$. Desse modo, a hipótese colinérgica, na qual as anormalidades sinápticas poderiam representar a causa da demência na doença de Alzheimer, admite uma melhor correlação entre o padrão e a severidade das alterações cognitivas em comparação às placas senis e $\mathrm{NFT}^{17}$.

Em relação às placas senis, quando a substância $A \beta$ encontra-se em altas concentrações, fibras amiloidais insolúveis são formadas no cérebro, as quais podem agregar-se ao zinco e ao cobre, agravando, assim, a toxicidade neuronal ${ }^{18}$. Vários estudos mostraram a correlação entre os metais e a biologia celular da APP e a neurodegeneração da doença de Alzheimer. Por exemplo, o cobre foi capaz de promover a agregação da substância $A \beta^{19}$, formando um complexo de alta afinidade $^{19}$, sendo que a neurotoxicidade desse composto depende do peróxido de hidrogênio 
formado pelo complexo $A \beta$-cobre in vitro ${ }^{19}$. Além disso, metais como o cobre, o zinco e o ferro foram encontrados em depósitos amiloidais de cérebros de pacientes portadores da doença de Alzheimer ${ }^{20}$. Nesse sentido, estudos mostraram que os quelantes do zinco e do cobre foram capazes de solubilizar essas fibras $A \beta$ em amostras de tecido post mortem de pacientes portadores da doença de Alzheimer ${ }^{21}$. Mais ainda, estes quelantes solubilizaram as placas amiloidais in vivo em um modelo animal da doença de Alzheimer ${ }^{22}$. Neste estudo, camundongos transgênicos tratados cronicamente com clioquinol (um antibiótico capaz de agregar-se ao cobre e de atravessar a barreira hematoencefálica) mostraram um declínio substancial na deposição amiloidal cerebral (aproximadamente 49\%). Esse efeito foi atribuído ao clioquinol, o qual atuou diretamente na redução do acúmulo de $A \beta^{22}$. Esse declínio foi associado a uma melhora significativa na saúde geral do animal e seu conseqüente aumento de peso, sem, no entanto, apresentar efeitos colaterais ${ }^{22}$.

A outra característica neuropatológica da doença de Alzheimer, os NFT, consistem em filamentos helicoidais procedentes da hiperfosforilação do citoesqueleto da proteína tau ${ }^{23}$. A hipótese da tau e dos NFT sugeriu que, na doença de Alzheimer, a função normal da proteína tau de estabilizar os microtúbulos neuronais foi prejudicada, e, mais ainda, esses microtúbulos de neurônios doentes foram gradualmente substituídos por NFT ${ }^{24}$.

\section{Aspectos genéticos}

O fator genético é considerado como preponderante na etiopatogenia da doença de Alzheimer. Além do componente genético, foram apontados como agentes etiológicos a toxicidade a agentes infecciosos, ao alumínio, a substâncias reativas de oxigênio (ROS) e a aminoácidos neurotóxicos, e a ocorrência de danos em microtúbulos e proteínas associadas ${ }^{25}$. É importante também salientar que esses agentes podem ainda atuar por dano direto no material genético, levando a uma mutação somática nos tecidos ${ }^{26}$.

Cerca de $1 / 3$ dos casos de doença de Alzheimer apresenta familiaridade e se comporta de acordo com um padrão de herança monogênica autossômica dominante. Esses casos, em geral, são de acometimento precoce, e famílias extensas têm sido periodicamente estudadas ${ }^{26}$. Os pacientes afetados pela doença de Alzheimer têm $50 \%$ de chance de ter filhos também afetados pela patologia 26 .

Mais ainda, uma associação entre a doença de Alzheimer e a síndrome de Down levou à descoberta do primeiro gene da doença de Alzheimer no cromossomo 21, o qual é o cromossomo extra envolvido na síndrome de Down. Indivíduos portadores da síndrome de Down apresentaram envelhecimento prematuro, e praticamente todos apresentaram doença de Alzheimer, clínica e neuropatologicamente confirmada, entre os 40 e 50 anos de idade ${ }^{27}$. Esse primeiro gene a ser identificado na doença de Alzheimer revelou-se o responsável pela APP, a qual acumula-se intensamente nas placas senis dos cérebros de pacientes portadores da doença de Alzheimer ${ }^{26}$.

Embora os vários fatores de risco genéticos - APP, PS1 e PS2 - estejam associados à doença de Alzheimer, a presença do alelo do gene apolipoproteína E do tipo 4 (apoE4) demonstrou uma maior associação ao número de placas senis e placas vasculares, além de uma redução da função colinérgica em cérebros de pacientes portadores dessa patologia 28 . O gene da APP localizado no cromossomo 21 e seu papel patogênico têm sido amplamente investigados em função do acúmulo da proteína $A \beta$ em cérebros de pacientes portadores da doença de Alzheimer ${ }^{26}$.

A presença do gene apoE4 no braço longo do cromossomo 19 esteve associada a fatores de risco significativos para a doença de Alzheimer ${ }^{29}$. A herança do gene da apoE4 eleva em até quatro vezes o risco para desenvolvimento da doença de Alzheimer, e esse risco aumenta ainda mais se o paciente herdar o alelo de ambos os pais ${ }^{24}$. 
Em relação às PS, mais de 40 mutações têm sido descritas para PS1 (cujo gene está localizado no cromossomo 14), as quais podem, subseqüentemente, resultar na forma esporádica da doença de Alzheimer ${ }^{24}$. Mutações nas PS 1 e 2 seletivamente aumentaram a produção de substância $A \beta$ em cultura de células e em cérebros de camundongos transgênicos e foram relacionadas ao desenvolvimento de formas esporádicas da doença de Alzheimer $^{30}$.

\section{Altos níveis plasmáticos de colesterol e o desenvolvimento da doença}

Um estudo epidemiológico demonstrou a relação direta entre altos níveis de colesterol sangüíneo e o aumento de risco de desenvolvimento da doença de Alzheimer ${ }^{31}$. Existem muitas evidências sugerindo uma forte relação entre a deterioração da homeostase lipídica cerebral, as alterações vasculares e a patogenia da doença de Alzheimer. Essas associações incluem: (1) reconhecimento de que um transportador do colesterol, a apoE4, atuou como o principal fator de risco genético para o desenvolvimento da doença de Alzheimer, tanto na forma familiar quanto na esporádica; (2) estudos epidemiológicos ligando os fatores de risco, como a hipertensão e altos níveis plasmáticos de colesterol, à demência; e (3) o efeito benéfico das drogas redutoras do colesterol, como as estatinas, no combate à doença de Alzheimer idiopática ${ }^{32}$. Evidências obtidas a partir de um estudo realizado em humanos indicaram que o tratamento à base de estatinas - lovastatina $\left(\right.$ Mevacor $\left.{ }^{\circledR}\right)$, sinvastatina $\left(\right.$ Zocor $\left.^{\circledR}\right)$, atorvastatina $\left(\right.$ Citalor $^{\circledR}$ ) e pravastatina (Pravacol ${ }^{\circledR}$ ) - conferiu proteção contra a forma esporádica da doença ${ }^{33}$. Outros estudos indicaram que o colesterol foi capaz de regular o processo proteolítico da APP, favorecendo a formação da substância $A \beta$, ao passo que uma redução dos níveis plasmáticos do colesterol foi capaz de reduzir a formação amiloidal ${ }^{34}$.

\section{A inflamação na doença de Alzheimer}

Mais recentemente, inúmeros estudos têm sido publicados questionando a terapia antiinflamatória, principalmente utilizando-se drogas não-esteroidais, como tratamento para a neuroinflamação ocasionada pelas ROS e espécies reativas de nitrogênio (RNS) e pela formação de radicais livres associados à doença de Alzheimer ${ }^{35}$.

As ROS e RNS produzidas intracelularmente e extracelularmente por vários mecanismos estão dentre os principais fatores de riscos intermediários, os quais iniciam e promovem a neurodegeneração na doença de Alzheimer esporádica ${ }^{36}$. Em um estudo, foi demonstrado que, através da ação das ROS, os lipídios tiveram sua conformação alterada, e, assim, ocorreu uma forte ligação entre os lipídios peroxidados, as enzimas antioxidantes, a presença de placas senis e os NFT em cérebros de pacientes portadores da doença de Alzheimer ${ }^{37}$. Além disso, a ação das ROS sobre o DNA, particularmente o radical hidroxila, levou à formação de marcadores biológicos ${ }^{38}$, os quais têm sido localizados nos NFT e placas senis de cérebros portadores da doença de Alzheimer ${ }^{39}$. O prejuízo do DNA induzido pelos radicais livres ou as modificações enzimáticas podem ter sido o gatilho inicial na apoptose presente na doença de Alzheimer ${ }^{40}$. Essas observações têm sido interpretadas como sugestão de que, em adição ao fato de o cérebro na doença de Alzheimer ser vulnerável ao aumento do estresse oxidativo, houve também uma redução na reparação desse prejuízo oxidativo, levando ao acúmulo de DNA alterados, os quais podem ser um importante fator na progressão da perda neuronal na doença de Alzheimer ${ }^{24}$.

Evidências bioquímicas têm demonstrado que a cadeia de transporte mitocondrial está afetada na doença de Alzheimer. Especificamente, a enzima citocromo C oxidase e o complexo IV da cadeia respiratória foram afetados nessa patologia ${ }^{41}$. Uma vez que foram demonstradas alterações na função das enzimas, e essas deficiências foram atribuídas às ROS, é possível que o dano oxidativo mitocondrial no terminal sináptico associado à idade possa ser extremamente importante, especialmente em relação ao declínio da memória e dos processos de aprendizagem ${ }^{24}$.

Em relação à neuroinflamação, algumas células especializadas no cérebro parecem estar 
envolvidas nas respostas inflamatórias, sendo as mais importantes os astrócitos e as células da micróglia ${ }^{24}$. Na doença de Alzheimer, o número de astrócitos reativos esteve elevado, e a expressão da PLA2 nessas células esteve aumentada, levando ao aumento da atividade da via inflamatória do ácido araquidônico/prostaglandina ${ }^{24}$. Os astrócitos foram capazes de liberar muitas moléculas pró-inflamatórias, como as interleucinas (IL), prostaglandinas (PG), leucotrienos, tromboxanos, fatores de coagulação, fatores de complemento e proteases, cujo processo foi similar ao que ocorreu às células da micróglia ${ }^{42}$. As células da micróglia ativadas também foram abundantes nos cérebros de pacientes portadores da doença de Alzheimer e produziram uma variedade de compostos neurotóxicos, incluindo radicais superóxidos, glutamato e óxido nítrico ${ }^{43}$. Mais ainda, a exposição da micróglia à substância $A \beta$ levou à liberação de fatores pró-inflamatórios, incluindo a interleucina-1 (IL-1), a interleucina-6 (IL-6), a interleucina-8 (IL-8) e o fator de necrose tumoral (TNF-a) ${ }^{44}$.

As células da glia do sistema nervoso central, como os astrócitos e a micróglia, expressaram várias subunidades neuronais dos receptores colinérgicos, incluindo $a 3, a 4, a 5, a 6, a 7, \beta 2$ e $\beta 4^{45}$. A subunidade a7 é o subtipo mais abundante encontrado nos astrócitos ${ }^{45}$. Um fato importante é que o nível da subunidade a7 mostrou-se elevado nos astrócitos de pacientes portadores da doença de Alzheimer, quando comparados a idosos não-doentes ${ }^{45}$. Uma possibilidade é que haja um mecanismo regulatório que auxilia a manutenção da produção de TNF-a e TNF- $\beta$ em níveis fisiológicos em astrócitos de cérebros de pacientes portadores da doença de Alzheimer ${ }^{42}$.

\section{A doença de Alzheimer e os antiinflamatórios}

Com base nas evidências de que os processos inflamatórios estejam envolvidos na patogênese da doença de Alzheimer, as pesquisas têm observado o uso de drogas antiinflamatórias nãoesteroidais e glicocorticóides esteroidais como opção de tratamento para pacientes portadores da doença de Alzheimer ${ }^{42}$ (Figura 1).

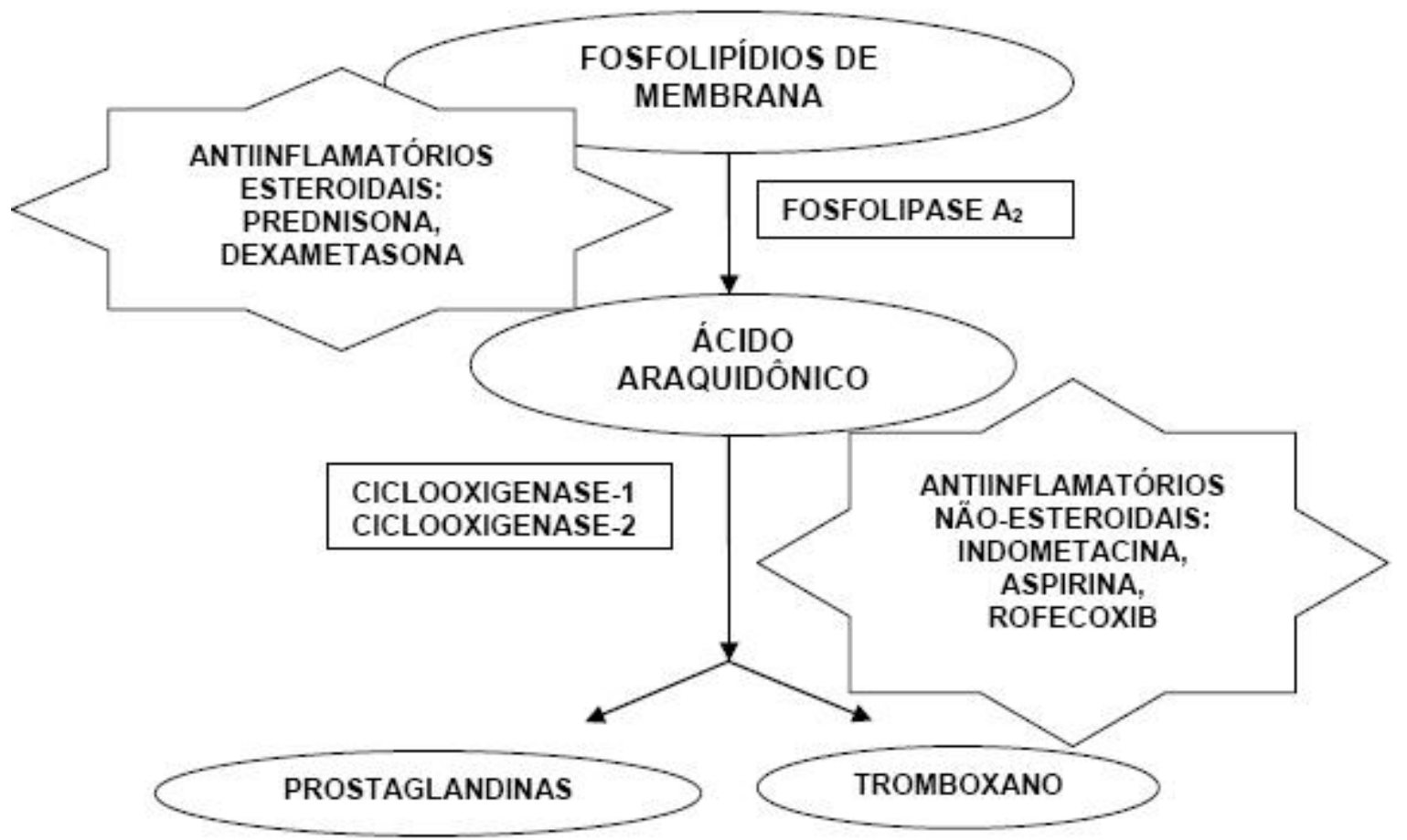

Figura 1 - Síntese de prostaglandinas e tromboxano a partir do ácido araquidônico e locais de ação dos antiinflamatórios esteroidais e não-esteroidais

Evidências epidemiológicas indicaram que as drogas antiinflamatórias não-esteroidais foram capazes de reduzir o risco de desenvolvimento da doença de Alzheimer ${ }^{46}$. Estudos post mortem 
têm demonstrado a habilidade das drogas antiinflamatórias não-esteroidais em reduzir a inflamação, a qual foi consistentemente observada no tecido cerebral de portadores da doença de Alzheimer ${ }^{47}$. Um possível modo de ação para a eficácia dos antiinflamatórios não-esteroidais seria o bloqueio específico da isoforma ciclooxigenase-2 (COX-2) no cérebro de pacientes portadores da doença de Alzheimer ${ }^{42}$. Estudos têm demonstrado que o RNA mensageiro (RNAm) da COX-2 esteve consideravelmente aumentado em áreas afetadas pela doença de Alzheimer ${ }^{48}$, sugerindo o envolvimento da COX-2 nessa doença. De fato, a aplicação de A $\beta$ (142) (5 microM) em culturas humanas de micróglia durante 8 horas induziu a expressão e 0 aumento da produção das citocinas pró-inflamatórias IL-6, IL-1 $\beta$, TNF-a, da COX-2 e IL-8 ${ }^{48}$.

O ibuprofeno $\left(\right.$ Advil $\left.^{\circledR}\right)$ e a indometacina (Indocid ${ }^{\circledR}$ ) - mas não o naproxeno (Naprosyn ${ }^{\circledR}$ ), o celecoxib (Celebra ${ }^{\circledR}$ ) ou o ácido acetilsalicílico (Aspirina ${ }^{\circledR}$ ) - demonstraram reduzir os níveis de $\mathrm{A} \beta$ acima de $80 \%$ em cultura de células ${ }^{49}$. Como nem todos os antiinflamatórios não-esteroidais apresentaram esse efeito, acredita-se que essa redução ocorreu por um processo independente da atividade antiinflamatória sobre a $\mathrm{COX}^{42}$.

Além disso, outro estudo também demonstrou que neurônios tratados com inibidores da COX-1, como o ibuprofeno e o ácido acetilsalicílico, foram mais resistentes aos efeitos da $A \beta$, em comparação aos neurônios tratados com inibidores da COX-250. O estudo também demonstrou uma redução na produção de PGE2 nos neurônios tratados com inibidores da COX-1 e COX-2. A indometacina foi capaz de suprimir a expressão de numerosos genes pró-inflamatórios em monócitos, além da micróglia ${ }^{51}$. A administração da indometacina também produziu neuroproteção por inibir a indução da COX-2, a qual foi capaz de potencializar a excitotoxicidade e aumentar a produção de radicais livres e fatores de necrose ${ }^{51}$.

Infelizmente, estudos clínicos com antiinflamatórios não-esteroidais em pacientes portadores da doença de Alzheimer não têm sido muito satisfatórios, desapontando especialmente quanto ao uso dos inibidores da COX-2 ${ }^{52}$. Um recente estudo randomizado, duplo-cego e placebocontrolado, determinando o efeito do inibidor seletivo da COX-2, o rofecoxib (Vioxx ${ }^{\circledR}$ ), e de um inibidor não-seletivo da COX, o naproxeno, em comparação ao grupo placebo, na progressão da doença de Alzheimer, demonstrou que esses antiinflamatórios não diminuíram o declínio cognitivo dos pacientes portadores da patologia suave a moderada ${ }^{53}$. Dentre as razões, os autores sugeriram a possibilidade de a neuropatologia estar muito avançada para a terapia com as drogas antiinflamatórias não-esteroidais surtirem efeito ${ }^{42}$. Outro estudo em ratos demonstrou que o rofecoxib reduziu a perda de neurônios colinérgicos e a resposta inflamatória cerebral induzida pelo ácido quisquálico ${ }^{54}$. Contudo, em um ensaio randomizado, duplo-cego e placebo controlado, o rofecoxib não reduziu o declínio cognitivo induzido pela doença de Alzheimer ${ }^{55}$.

Um estudo, a fim de determinar o efeito do ácido acetilsalicílico (inibidor não-seletivo da COX) sobre a agregação da $A \beta$ e sobre a produção de APP, demonstrou que essa droga não foi capaz de modificar os níveis de $A \beta$ e APP no soro e no plasma ${ }^{56}$, sugerindo que a COX não exerceu um papel importante na secreção de APP.

Um dos fatores de prevenção quanto ao uso dos antiinflamatórios não-esteroidais para redução do risco de desenvolvimento da doença de Alzheimer é a toxicidade associada a esses medicamentos ${ }^{42}$. As drogas antiinflamatórias não-esteroidais foram reconhecidas por causar problemas gastrointestinais, renais, hematológicos, cardiovasculares e sobre o sistema nervoso central $^{42}$, sendo que a população idosa, a qual faria uso desses medicamentos, seria mais suscetível aos efeitos tóxicos.

Quanto aos antiinflamatórios esteroidais, os glicocorticóides foram considerados agentes antiinflamatórios potentes, atuaram na regulação da transcrição das moléculas inflamatórias, inibindo a ação das enzimas que mediariam a produção de PG, a COX, e reduziram a expressão de citocinas, as quais foram pró-inflamatórias ${ }^{47}$. Entretanto, a evidência epidemiológica na qual os antiinflamatórios esteroidais (glicocorticóides sintéticos) seriam benéficos no tratamento da 
doença de Alzheimer foi relativamente fraca ${ }^{57}$. Um estudo randomizado e placebo-controlado, conduzido a fim de determinar se o tratamento com prednisona (Meticorten ${ }^{\circledR}$ ) diminuiria 0 declínio cognitivo em pacientes portadores da doença de Alzheimer, demonstrou que não houve diferença significativa entre o grupo tratado e o grupo controle ${ }^{58}$.

\title{
TRATAMENTO FARMACOLÓGI CO DA DOENÇA DE ALZHEI MER
}

\author{
Tratamento dos sintomas cognitivos
}

Grandes esforços têm sido realizados para a compreensão e tratamento da doença de Alzheimer; entretanto, a terapia atual está longe de ser satisfatória. De fato, embora o tratamento realizado através da administração de inibidores da enzima acetilcolinesterase (AChE) tenha consistentemente demonstrado eficácia sintomática e redução na progressão da patologia, esses medicamentos produziram algum tipo de melhora em aproximadamente 30$40 \%$ dos pacientes portadores da doença de Alzheimer leve a moderada ${ }^{59}$.

Os inibidores da acetilcolinesterase (tacrina, rivastigmina, donepezil, galantamina) alteram a função colinérgica central ao inibir as enzimas que degradam a acetilcolina (enzimas acetilcolinesterase e butirilcolinesterase), aumentando, assim, a capacidade da acetilcolina de estimular os receptores nicotínicos e muscarínicos cerebrais ${ }^{60}$. Desde a introdução desses medicamentos na prática clínica, os inibidores da AChE constituem o tratamento sintomático de escolha para a doença de Alzheimer ${ }^{60}$.

A rivastigmina (Exelon ${ }^{\circledR}$ ), atualmente um dos medicamentos mais utilizados no tratamento da doença de Alzheimer, foi capaz de inibir tanto a enzima acetilcolinesterase quanto a butirilcolinesterase, apresentando, assim, maior eficácia quanto ao aumento dos níveis cerebrais de acetilcolina ${ }^{60}$. Entretanto, esse medicamento causou vários efeitos colinérgicos adversos quando a dose foi elevada abruptamente ${ }^{60}$. Mais ainda, esse medicamento apresentou efeitos gastrointestinais adversos, associados ao aumento de peso ${ }^{60}$.

Outro estudo envolvendo um inibidor seletivo da acetilcolinesterase, o donepezil (Aricept ${ }^{\circledR}$ ), demonstrou que o tratamento crônico por 1 ano com esse medicamento foi associado a uma redução de $38 \%$ no declínio funcional dos pacientes portadores da doença de Alzheimer, quando comparados ao grupo placebo61.

A tacrina $\left(\right.$ Cognex $\left.{ }^{\circledR}\right)$ foi o primeiro inibidor reversível da acetilcolinesterase a ser utilizado no tratamento da doença de Alzheimer (inibidor de primeira geração). Entretanto, esse medicamento apresentou duas formas principais de toxicidade. Primeiramente, a tacrina apresentou hepatoxicidade, levando ao aumento das transaminases hepáticas, resultando em hepatite medicamentosa ${ }^{62}$ e provocando a retirada da medicação em muitos pacientes. De fato, mais de $90 \%$ dos casos envolvendo a hepatite medicamentosa na doença de Alzheimer ocorreram nas primeiras 12 semanas de tratamento com a tacrina ${ }^{62}$. Recentemente, estudos têm focado na síntese e avaliações de congêneres da tacrina, os quais foram mais potentes e seletivos que o próprio medicamento original ${ }^{63}$.

A galantamina (Reminy $l^{\circledR}$ ) também é um anticolinesterásico utilizado no tratamento da doença de Alzheimer. Esse medicamento possui um duplo mecanismo de ação, o qual, além de inibir a acetilcolinesterase, também foi capaz de modular alostericamente os receptores nicotínicos ${ }^{64}$. 0 papel dos receptores nicotínicos na cognição é bastante conhecido, entretanto, o significado 
clínico da modulação nicotínica no tratamento da doença de Alzheimer não está completamente elucidado. Os receptores nicotínicos pré-sinápticos controlam a liberação de neurotransmissores, os quais são importantes para a memória e para o humor. Nesse sentido, foi verificado que o bloqueio dos receptores nicotínicos prejudicou a cognição ${ }^{65}$. Por outro lado, a ligação da galantamina com os subtipos de receptores nicotínicos melhorou a função cognitiva e a memória ${ }^{65}$.

A huperzina A, um alcalóide extraído da planta Huperzia serrata, atuou como um inibidor potente, específico e reversível da acetilcolinesterase, cuja administração demonstrou melhora significativa na memória de pacientes idosos e de pacientes portadores da doença de Alzheimer, associada a mínimos efeitos colinérgicos periféricos e ausência da hepatoxicidade induzida pela tacrina ${ }^{66}$.

A fim de atuar em mais de um fator relacionado ao desenvolvimento da doença de Alzheimer, a combinação de compostos poderia ser útil para o tratamento dessa patologia. Nesse sentido, antioxidantes lipofílicos, como o a-tocoferol, foram utilizados com algum sucesso em alguns estudos clínicos envolvendo pacientes portadores da doença de Alzheimer ${ }^{24}$. Outra terapia que tem sido avaliada é a utilização do fator neurotrófico derivado do cérebro, o qual estimula o crescimento do gânglio parassimpático no sistema nervoso periférico, da substância negra, do hipocampo e dos neurônios motores no sistema nervoso central ${ }^{24}$.

Há ainda um crescente interesse no uso de antioxidantes polifenólicos, a fim de reverter o declínio associado à idade e as deficiências cognitivas e motores dos pacientes portadores da doença de Alzheimer ${ }^{24}$. Um estudo in vitro e in vivo envolvendo 2,4-dinitrofenol e 3-nitrofenol demonstrou que esses nitrofenóis foram capazes de inibir a agregação amiloidal, além de provocar a desagregação das fibras amiloidais já formadas ${ }^{67}$. Esses efeitos foram acompanhados por uma proteção contra a toxicidade induzida por $A \beta$ em culturas primárias de neurônios hipocampais ${ }^{67}$. Mais ainda, experimentos utilizando microinjeções desses nitrofenóis mostraram que essas substâncias foram capazes de inibir a deposição amiloidal em cérebros de ratos $^{67}$.

Em outros experimentos, a taurina tem demonstrado proteção contra a excitotoxicidade induzida pelo glutamato em outros modelos celulares, embora os mecanismos para essa proteção ainda não tenham sido completamente esclarecidos ${ }^{68}$.

Outros trabalhos têm demonstrado as propriedades inibitórias de algumas substâncias na oligomerização $A \beta$. Em um estudo in vitro, alguns componentes, como o 2-amino-4-clorofenol, o 4-aminofenol, o ácido 3-4-dihidroxibenzóico, demonstraram atividade antiamiloidogênica, a qual inibiu a formação de oligômeros $A \beta$, bem como de fibras amiloidais, e ainda reduziram as fibras amiloidais já formadas ${ }^{69}$. Mais ainda, esses componentes bloquearam completamente a neurotoxicidade induzida pela $A \beta$ em cultura de neurônios hipocampais de ratos $^{69}$.

A estratégia mais estudada para o tratamento da doença de Alzheimer tem sido bloquear a maquinaria proteolítica, a qual produz a substância $A \beta$. Essa estratégia pode ser acompanhada pela redução da formação de APP ou pela inibição da proteólise da APP para a formação da $A \beta^{24}$. A inibição das vias $\beta$ e $\gamma$-secretase (via amiloidogênica da clivagem proteolítica da APP) e a estimulação da via a-secretase têm sido as estratégias mais promissoras para a neuroproteção na doença de Alzheimer ${ }^{24}$.

\section{Tratamento dos sintomas comportamentais}

Embora haja evidências clínicas suficientes para sua indicação, as avaliações da eficácia do tratamento antidepressivo em pacientes portadores da doença de Alzheimer são limitadas ${ }^{5}$. Boa parte dos conhecimentos desse tratamento vem do estudo de casos individuais e de extrapolações de estudos com pacientes geriátricos deprimidos com ausência de demência 5 . Determinados sintomas podem ser facilmente confundidos com depressão, incluindo perda de 
interesse em atividades diárias e cuidados pessoais, redução da iniciativa e das interações sociais ou da expressão facial, redução da entonação verbal e das respostas emocionais ${ }^{5}$. Na depressão, contudo, tais queixas são geralmente associadas à disforia, ideação depressiva e sintomas vegetativos ${ }^{5}$.

O controle da depressão em pacientes portadores da doença de Alzheimer pode proporcionar, indiretamente, melhora substancial na cognição ${ }^{5}$. Evidentemente, sintomas cognitivos além daqueles induzidos pela depressão não se beneficiam do tratamento antidepressivo. Além disso, determinados antidepressivos podem exercer efeito negativo sobre a cognição, conforme sugerido por um estudo envolvendo a imipramina (Tofranil ${ }^{\circledR}$ ) e a amitriptilina (Tryptanol ${ }^{\circledR}$ ), no qual a piora dos sintomas cognitivos foi atribuída aos efeitos anticolinérgicos da medicação ${ }^{70}$. Knegtering et al. ${ }^{71}$ revisaram os efeitos dos antidepressivos no funcionamento cognitivo de pacientes idosos, concluindo que a ação sedativa de alguns psicofármacos pode comprometer o desempenho em provas que requeiram concentração e manutenção do tônus atencional, enquanto drogas com ação anticolinérgica podem afetar diretamente a memória. Tais efeitos podem se manter ao longo do tratamento, porém podem passar despercebidos devido à melhora do humor e do estado geral do paciente ${ }^{5}$.

Os inibidores da recaptação de serotonina são usualmente as drogas de primeira escolha no tratamento da depressão em idosos com ou sem demência ${ }^{72}$. Um estudo placebo-controlado, utilizando citalopram na dose de $10-30 \mathrm{mg} / \mathrm{dia}$ em pacientes idosos deprimidos com e sem demência, demonstrou melhora significativa nos escores para depressão, labilidade emocional, ansiedade, agitação e interação social no grupo tratado ${ }^{73}$. Os efeitos mais comuns dos inibidores da recaptação de serotonina são as alterações gastrointestinais (náuseas, vômitos e diarréias), insônia, inquietação e, mais raramente, a hiponatremia ${ }^{5}$. Interações medicamentosas entre drogas de uso clínico e alguns inibidores da recaptação de serotonina exigem atenção, especialmente a fluoxetina (Prozac ${ }^{\circledR}$ ) e a fluvoxamina (Luvox $\left.{ }^{\circledR}\right)^{5}$. A sertralina (Zoloft ${ }^{\circledR}$ ) e o citalopram (Cipramil ${ }^{\circledR}$ ) devem ser preferidos, por possuírem meia-vida plasmática mais curta, pouca atividade de seus metabólitos, baixo risco de interações medicamentosas e relação dose/resposta linear ${ }^{5}$.

Os antidepressivos tricíclicos não devem ser utilizados como primeira opção terapêutica em pacientes portadores da doença de Alzheimer. Seus efeitos colaterais mais importantes em idosos acarretam hipotensão postural, retardo miccional em alguns casos, obstipação intestinal, visão turva e alterações cardíacas ${ }^{5}$. Efeitos prejudiciais sobre a cognição podem advir de suas ações anticolinérgicas e sedativas ${ }^{72}$.

A trazodona (Donaren ${ }^{\circledR}$ ), cuja ação é predominantemente serotoninérgica, associada a poucos efeitos anticolinérgicos, possui uma ação sedativa importante ${ }^{5}$.

Os inibidores da monoamina oxidase (MAO), como a fenelzina (Nardil ${ }^{\circledR}$ ) e a tranilcipromina (Parnate ${ }^{\circledR}$ ), apresentaram poucas propriedades anticolinérgicas, porém seu uso deve ser indicado apenas nos casos refratários às demais opções, pela ocorrência de hipotensão postural (risco de quedas e fraturas) ou crises hipertensivas por falhas dietéticas ${ }^{5}$. A monitorização da hipotensão postural é indispensável, e os cuidados devem ser redobrados em relação à dieta alimentar e interação a outros medicamentos ${ }^{5}$.

Embora extremamente eficaz e segura no tratamento da depressão grave em idosos que apresentam demência ${ }^{74}$, há poucos estudos relacionando os efeitos terapêuticos da eletroconvulsoterapia (ECT) em pacientes portadores da doença de Alzheimer. A ECT pode ser eficaz em casos selecionados, porém deve ser indicada apenas para os pacientes que apresentarem depressão grave, pacientes refratários ou intolerantes às demais terapias farmacológicas ${ }^{5}$. A necessidade de resposta rápida, como na presença de idéias suicidas ou em casos de desnutrição grave, apóia a indicação de ECT em idosos que apresentam demência 5 . 
Além dos medicamentos antidepressivos, no que tange às anormalidades comportamentais apresentadas na doença de Alzheimer, medicamentos antipsicóticos, como o haloperidol (Haldol ${ }^{\circledR}$ ), e os benzodiazepínicos, como o diazepam (Valium ${ }^{\circledR}$ ), têm sido utilizados no intuito de facilitar os cuidados com o paciente, especialmente reduzindo as alucinações, a agressividade, os distúrbios de humor, a anedonia, a apatia e a disforia, além de outros comportamentos que ocorrem com a evolução da patologia 4 .

A literatura a respeito do tratamento da apatia é ainda mais escassa. Drogas com ação dopaminérgica, tais como os psicoestimulantes - dextroanfetamina (Dexedrine ${ }^{\circledR}$ ), metilfenidato (Ritalina ${ }^{\circledR}$ ) -, amantadina (Mantidan ${ }^{\circledR}$ ), bromocriptina (Parlodel ${ }^{\circledR}$ ) e bupropiona (Zyban ${ }^{\circledR}$ ) são úteis na terapia farmacológica da apatia grave $^{5}$. Psicoestimulantes podem provocar taquicardias, hipertensão, inquietação motora, agitação, distúrbios do sono e supressão do apetite $^{5}$. A bromocriptina esteve associada a sintomas psicóticos, confusão mental e, algumas vezes, a discinesias ${ }^{5}$, enquanto a amantadina acarretou efeitos anticolinérgicos e delírios ${ }^{5}$.

Especialmente quando associadas à demência avançada, essas alterações comportamentais presentes em portadores da doença de Alzheimer podem ser dificilmente caracterizadas, principalmente na presença de distúrbios de linguagem e comunicação ou quando as demais funções cognitivas estiverem gravemente comprometidas ${ }^{5}$.

\section{CONCLUSÃO}

Os resultados de alguns estudos epidemiológicos, os quais utilizaram agentes antiinflamatórios, sugerem que a neuroinflamação possa exercer um papel inicial na patogênese da doença de Alzheimer; porém, estudos clínicos, especialmente envolvendo inibidores seletivos da COX-2, têm sido desapontadores.

Mais ainda, outros fatores, como, por exemplo, o sistema complemento para receptores nicotínicos, o qual está implicado no processo inflamatório associado à doença de Alzheimer, demonstram que existem ainda muitos mecanismos relacionados à patologia que precisam ser compreendidos.

Outros fatores a serem considerados são que muitos dos participantes do processo inflamatório, como a micróglia e os astrócitos, podem ter funções tanto neuroprotetoras quanto neurodegenerativas, tornando seus papéis difíceis de serem determinados no processo da doença.

Embora muitos estudos tenham contribuído para elucidar os mecanismos fisiopatológicos da doença de Alzheimer, a perda neuronal seletiva ainda não foi totalmente compreendida. Mais ainda, a busca desses mecanismos tem resultado direto no desenvolvimento de novas drogas para o tratamento dessa patologia, sendo que a investigação de novos agentes medicamentosos que possam retardar ou mesmo bloquear a evolução da doença constitui o objetivo e o desafio para muitos neurocientistas. 


\section{AGRADECI MENTOS}

Nossos sinceros agradecimentos ao Dr. Roberto Andreatini, professor do Departamento de Farmacologia da Universidade Federal do Paraná, por suas valiosas críticas e sugestões na leitura deste trabalho.

\section{REFERÊNCI AS}

1. Zhao Q, Tang XC. Effects of huperzine A on an acetylcholinesterase isoforms in vitro: comparison with tacrine, donepezil, rivastigmine and physostigmine. Eur J Pharmacol. 2002;455 $(2-3): 101-7$.

2. Janus C, Westaway D. Transgenic mouse models of Alzheimer's disease. Physiol Behav. $2001 ; 73(5): 873-86$.

3. Lindeboom J, Weinstein $\mathrm{H}$. Neuropsychology of cognitive ageing, minimal cognitive impairment, Alzheimer's disease, and vascular cognitive impairment. Eur J Pharmacol. $2004 ; 490(1-3): 83-6$.

4. Raskind MA. Alzheimer's disease: treatment of noncognitive behavioural abnormalities. In: Bloom FE, Kupper DJ. Psychopharmacology: the fourth generation of progress. New York: Raven Press; 1995. p. 1427-35.

5. Forlenza OV. Transtornos depressivos na doença de Alzheimer: diagnóstico e tratamento. Rev Bras Psiquiatr. 2000;22(2):87-95.

6. Wragg RE, Jeste DV. Overview of depression and psychosis in Alzheimer's disease. Am J Psychiatry. 1989;146(5):577-87.

7. Eastwood R, Reisberg B. Mood and behaviour. In: Gauthier S, editor. Clinical diagnosis and management of Alzheimer's disease. London: Martin Dunitz; 1996. p. 175-90.

8. Selkoe D. Alzheimer's disease: genes, proteins, and therapy. Physiol Rev. 2001;81(2):74166.

9. Hardy J, Selkoe DJ. The amyloid hypothesis of Alzheimer's disease: progress and problems on the road to therapeutics. Science. 2002;297(5580):353-6.

10. Bartus RT, Emerich DF. Cholinergic markers in Alzheimer disease. JAMA. 1999;282 (23):2208-9.

11. Auld DS, Kornecook TJ, Bastianetto S, Quirion R. Alzheimer's disease and the basal forebrain cholinergic system: relations to beta-amyloid peptides, cognition and treatment strategies. Prog Neurobiol. 2002;68(3):209-45.

12. Gattaz WF, Forlenza OV, Talib LL, Barbosa NR, Bottino CM. Platelet phospholipase A2 activity in Alzheimer's disease and mild cognitive impairment. J Neural Transm. 2004;111 (5):591-601. 
13. Gattaz WF, Maras A, Cairns NJ, Levy R, Forstl H. Decreased phospholipase A2 activity in Alzheimer brains. Biol Psychiatry. 1995;37(1):13-7.

14. Gattaz WF, Cairns NJ, Levy R, Forstl H, Braus DF, Maras A. Decreased phospholipase A2 activity in the brain and in platelet of patients with Alzheimer's disease. Eur Arch Psychiatry Clin Neurosci. 1996;246(3):129-31.

15. Gattaz FW, Kollisch M, Thuren T, Virtanen JA, Kinnunen PK. Increased plasma phospholipase A2 activity in schizophrenic patients: reduction after neuroleptic therapy. Biol Psychiatry. 1987;22(4):421-6.

16. Fuentealba RA, Farias G, Scheu J, Bronfman M, Marzolo MP, Inestrosa NC. Signal transduction during amyloid-beta-peptide neurotoxicity: role in Alzheimer disease. Brain Res Brain Res Rev. 2004;47(1-3):275-89.

17. Braak $\mathrm{E}$, Braak H. Alzheimer's disease: transiently developing dendritic changes in pyramidal cells of sector CA1 of the Ammon's horn. Acta Neuropathol (Berl). 1997;93(4):323-5.

18. Huang X, Cuajungco MP, Atwood CS, Hartshorn MA, Tyndall JD, Hanson GR, et al. Cu(II) potentiation of Alzheimer abeta neurotoxicity. Correlation with cell-free hydrogen peroxide production and metal reduction. J Biol Chem. 1999;274(52):37111-6.

19. Atwood CS, Moir RD, Huang X, Scarpa RC, Bacarra NM, Romano DM, et al. Dramatic aggregation of Alzheimer abeta by $\mathrm{Cu}(\mathrm{II})$ is induced by conditions representing physiological acidosis. J Biol Chem. 1998;273(21):12817-26.

20. Lowell MA, Robertson JD, Teesdale WJ, Campbell JL, Markesbery WR. Copper, iron and zinc in Alzheimer's disease senile plaques. J Neurol Sci. 1998;158(1):47-52.

21. Cherny RA, Legg JT, McLean CA, Fairlie DP, Huang X, Atwood CS, et al. Aqueous dissolution of Alzheimer's disease abeta amyloid deposits by biometal depletion. J Biol Chem. 1999;274 (33):23223-8.

22. Cherny RA, Atwood CS, Xilinas ME, Gray DN, Jones WD, McLean CA, et al. Treatment with a copper-zinc chelator markedly and rapidly inhibits beta-amyloid accumulation in Alzheimer's disease transgenic mice. Neuron. 2001;30(3):665-76.

23. About a peculiar disease of the cerebral cortex. By Alois Alzheimer, 1907 (translated by L. Jarvik and H. Greenson). Alzheimer Dis Assoc Disord. 1987;1(1):3-8.

24. Parihar MS, Hemnani T. Alzheimer's disease pathogenesis and therapeutic interventions. J Clin Neurosci. 2004;11(5):456-67.

25. Mestel R. Putting prions to the test. Science. $1996 ; 273(5272): 184-9$.

26. Smith MAC. Doença de Alzheimer. Rev Bras Psiquiatr. 1999;21(Suppl 2):3-7.

27. Malamud N. Neuropathology of organic brain syndromes associated with aging. In: Gaitz CM, editor. Aging and the brain. New York: Plenum Press; 1972. p. 63-87.

28. Poirier J, Delisle MC, Quirion R, Aubert I, Farlow M, Lahiri D, et al. Apolipoprotein E4 allele as a predictor of cholinergic deficits and treatment outcome in Alzheimer disease. Proc Natl Acad Sci USA. 1995;92(26):12260-4.

29. Kosunen O, Talasniemi S, Lehtovirta M, Heinonen O, Helisalmi S, Mannermaa A, et al. Relation of coronary atherosclerosis and apolipoprotein $\mathrm{E}$ genotypes in Alzheimer patients. Stroke. $1995 ; 26(5): 743-8$. 
30. Citron M. Secretases as targets for the treatment of Alzheimer's disease. Mol Med Today. 2000;6(10):392-7.

31. Kivipelto M, Helkala EL, Hanninen T, Laakso MP, Hallikainen M, Alhainen K, et al. Midlife vascular risk factors and late-life mild cognitive impairment: a population-based study.

Neurology. 2001;56(12):1683-9.

32. Poirier J. Apolipoprotein $\mathrm{E}$ and cholesterol metabolism in the pathogenesis and treatment of Alzheimer's disease. Trends Mol Med. 2003;9(3):94-101.

33. Jick H, Zornberg GL, Jick SS, Seshadri S, Drachman DA. Statins and the risk of dementia. Lancet. $2000 ; 356(9242): 1627-31$.

34. Fassbender K, Simons M, Bergmann C, Stroick M, Lutjohann D, Keller P, et al. Simvastatin strongly reduces levels of Alzheimer's disease beta-amyloid peptides abeta 42 and abeta 40 in vitro and in vivo. Proc Natl Acad Sci USA. 2001;98(10):5856-61.

35. Moore AH, O'Banion MK. Neuroinflammation and anti-inflammatory therapy for Alzheimer's disease. Adv Drug Deliv Rev. 2002;54(12):1627-56.

36. Perry G, Taddeo MA, Nunomura A, Zhu X, Zenteno-Savin T, Drew KL, et al. Comparative biology and pathology of oxidative stress in Alzheimer and other neurodegenerative diseases: beyond damage and response. Comp Biochem Physiol C Toxicol Pharmacol. 2002;133(4):50713.

37. Lovell MA, Ehmann WD, Butler SM, Markesbery WR. Elevated thiobarbituric acid-reactive substances and antioxidant enzyme activity in the brain in Alzheimer's disease. Neurology. $1995 ; 45(8): 1594-601$.

38. Dizdaroglu M. Oxidative damage to DNA in mammalian chromatin. Mutat Res. $1992 ; 275(3-$ 6):331-42.

39. Smith MA, Sayre LM, Vitek MP, Monnier VM, Perry G. Early AGEing and Alzheimer's. Nature. $1995 ; 374(6520): 316$.

40. Stefanis L, Park DS, Friedman WJ, Greene LA. Greene, caspase-dependent and independent death of camptothecin-treated embryonic cortical neurons. J Neurosci. 1999;19(15):6235-47.

41. Parker WD Jr., Parks J, Filley CM, Kleinschmidt-DeMasters BK. Electron transport defects in Alzheimer's disease brain. Neurology. 1994;44(6):1090-6.

42. Tuppo EE, Arias HR. The role of inflammation in Alzheimer's disease. Int J Biochem Cell Biol. $2005 ; 37(2): 289-305$.

43. Brown GC, Bal-Price A. Inflammatory neurodegeneration mediated by nitric oxide, glutamate and mitochondria. Mol Neurobiol. 2003;27(3):325-55.

44. Rogers J, Lue LF. Microglial chemotaxis, activation, and phagocytosis of amyloid betapeptide as linked phenomena in Alzheimer's disease. Neurochem Int. 2001;39(5-6):333-40.

45. Teaktong T, Graham A, Court J, Perry R, Jaros E, Johnson M, et al. Alzheimer's disease is associated with a selective increase in alpha7 nicotinic acetylcholine receptor immunoreactivity in astrocytes. Glia. 2003;41(2):207-11.

46. Hoozemans JJ, Veerhuis R, Rozemuller AJ, Eikelenboom P. Non-steroidal anti-inflammatory drugs and cyclooxygenase in Alzheimer's disease. Curr Drug Targets. 2003;4(6):461-8. 
47. Mackenzie IR. Postmortem studies of the effect of anti-inflammatory drugs on Alzheimertype pathology and associated inflammation. Neurobiol Aging. 2001;22(6):819-22.

48. Ho L, Pieroni C, Winger D, Purohit DP, Aisen PS, Pasinetti GM. Regional distribution of cyclooxygenase-2 in the hippocampal formation in Alzheimer's disease. J Neurosci Res. 1999;57 (3):295-303.

49. Weggen S, Eriksen JL, Das P, Sagi SA, Wang R, Pietrzik CU, et al. A subset of NSAIDs lower amyloidogenic abeta42 independently of cyclooxygenase activity. Nature. $2001 ; 414(6860): 212-$ 6.

50. Bate C, Veerhuis R, Eikelenboom P, Williams A. Neurones treated with cyclo-oxygenase-1 inhibitors are resistant to amyloid-beta1-42. Neuroreport. 2003;14(16):2099-103.

51. Kelley KA, Ho L, Winger D, Freire-Moar J, Borelli CB, Aisen PS, et al. Potentiation of excitotoxicity in transgenic mice overexpressing neuronal cyclooxygenase-2. Am J Pathol. 1999;155(3):995-1004.

52. Aisen PS. The potential of anti-inflammatory drugs for the treatment of Alzheimer's disease. Lancet Neurol. 2002;1(5):279-84.

53. Aisen PS, Schafer KA, Grundman M, Pfeiffer E, Sano M, Davis KL, et al. Effects of rofecoxib or naproxen vs placebo on Alzheimer disease progression: a randomized controlled trial. JAMA. 2003;289(21):2819-26.

54. Scali C, Giovannini MG, Prosperi C, Bellucci A, Pepeu G, Casamenti F. The selective cyclooxygenase-2 inhibitor rofecoxib suppresses brain inflammatory and protects cholinergic neurons from excitotoxic degeneration in vivo. Neuroscience. 2003;117(4):909-19.

55. Reines SA, Block GA, Morris JC, Liu G, Nessly ML, Lines CR, et al. Rofecoxib: no effect on Alzheimer's disease in a 1-year, randomized, blinded, controlled study. Neurology. 2004;62 (1):66-71.

56. Skovronsky DM, Lee VM, Pratico D. Amyloid precursor protein and amyloid beta peptide in human platelets. Role of cyclooxygenase and protein kinase C. J Biol Chem. 2001;276 (20):17036-43.

57. Hull M, Lieb K, Fiebich BL. Pathways of inflammatory activation in Alzheimer's disease: potential targets for disease modifying drugs. Curr Med Chem. 2002;9(1):83-8.

58. Aisen PS, Davis KL, Berg JD, Schafer K, Campbell K, Thomas RG, et al. A randomized controlled trial of prednisone in Alzheimer's disease: Alzheimer's disease cooperative study. Neurology. 2000;54(3):588-93.

59. Kihara T, Sawada H, Nakamizo T, Kanki R, Yamashita H, Maelicke A, et al. Galantamine modulates nicotinic receptor and blocks abeta-enhanced glutamate toxicity. Biochem Biophys Res Commun. 2004;325(3):976-82.

60. Grossberg GT. Cholinesterase inhibitors for the treatment of Alzheimer's disease: getting on and staying on. Curr The Res. 2003;64(4):216-35.

61. Fergusson E, Howard R. Donepezil for the treatment of psychosis in dementia with Lewy bodies. Int J Geriatr Psychiatry. 2000;15(3):280-1.

62. Almeida OP. Tratamento da doença de Alzheimer: avaliação crítica sobre o uso de anticolinesterásicos. Arq Neuropsiquiatr. 1998;56(3B):688-96. 
63. Shao D, Zou C, Luo C, Tang X, Li Y. Synthesis and evaluation of tacrine-E2020 hybrids as acetylcholinesterase inhibitors for the treatment of Alzheimer's disease. Bioorg Med Chem Lett. 2004; 14(18):4639-42.

64. Maelicke A, Albuquerque EX. Allosteric modulation of nicotinic acetylcholine receptors as a treatment strategy for Alzheimer's disease. Eur J Pharmacol. 2000;393(1-3):165-70.

65. Levin ED, Rezvani AH. Development of nicotinic drug therapy for cognitive disorders. Eur J Pharmacol. 2000;393(1-3):141-6.

66. Zhang Z, Wang X, Chen Q, Shu L, Wang J, Shan G. Clinical efficacy and safety of huperzine Alpha in treatment of mild to moderate Alzheimer disease, a placebo-controlled, double-blind, randomized trial. Zhonghua Yi Xue Za Zhi. 2002;82(14):941-4.

67. De Felice FG, Houzel JC, Garcia-Abreu J, Louzada PR Jr, Afonso RC, Meirelles MN, et al. Inhibition of Alzheimer's disease beta-amyloid aggregation, neurotoxicity, and in vivo deposition by nitrophenols: implications for Alzheimer's therapy. FASEB J. 2001;15(7):1297-9.

68. Louzada PR, Lima AC, Mendonça-Silva DL, Noel F, De Mello FG, Ferreira ST. Taurine prevents the neurotoxicity of beta-amyloid and glutamate receptors agonists: activation of GABA receptor and possible implications for Alzheimer's disease and other neurological disorders. FASEB J. 2004;18(3):511-8.

69. De Felice FG, Vieira MN, Saraiva LM, Figueroa-Villar JD, Garcia-Abreu J, Liu R, et al. Targeting the neurotoxic species in Alzheimer's disease: inhibitors of Abeta oligomerization. FASEB J. 2004;18(12):1366-72.

70. Reifler BV, Teri L, Raskind M, Veith R, Barnes R, White E, et al. Double-blind trial of imipramine in Alzheimer's disease patients with and without depression. Am J Psychiatry. $1989 ; 146(1): 45-9$.

71. Knegtering $A H$, Eijck $M$, Huijsman A. Effects of antidepressants on cognitive functioning of elderly patients. Drugs Aging. 1994;5(3):192-9.

72. Tune LE. Depression and Alzheimer's disease. Depress Anxiety. 1998;8 Suppl 1:91-5.

73. Nyth AL, Gottfries CG, Lyby K, Smedegaard-Andersen L, Gylding-Sabroe J, Kristensen M, et al. A controlled multicenter clinical study of citalopram and placebo in elderly depressed patients with and without concomitant dementia. Acta Psychiatr Scand. 1992;86(2):138-45.

74. Prudic J, Sackeim HA. Electroconvulsive therapy for mood disorders. Curr Rev Mood Anxiety Disord. 1997;1(3):177-88.

\section{团 Correspondência}

Maria A. B. F. Vital

Universidade Federal do Paraná, Setor de Ciências Biológicas, Departamento de Farmacologia

Av. Francisco H. dos Santos, s/no

Cx. Postal 19031

CEP 81531-990, Curitiba, PR

Fax: (41) 3266-2042, (41) 3361-1720

E-mail: vital@ufpr.br 
Recebido em 04/07/2005.

Aceito em 04/01/2007. 